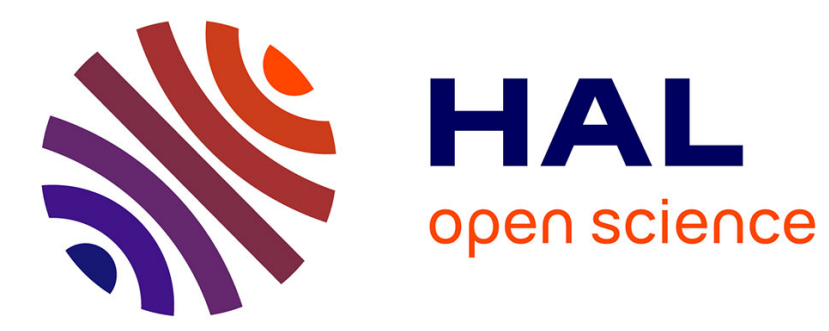

\title{
Nouvelle structure de capteur à courants de Foucault et algorithme associé pour la mesure des propriétés électrique et magnétique d'un métal \\ Minh-Quang Lê, Dominique Placko
}

\section{- To cite this version: \\ Minh-Quang Lê, Dominique Placko. Nouvelle structure de capteur à courants de Foucault et algo- rithme associé pour la mesure des propriétés électrique et magnétique d'un métal. Journal de Physique III, 1995, 5 (6), pp.807-824. 10.1051/jp3:1995162 • jpa-00249348}

\section{HAL Id: jpa-00249348 https://hal.science/jpa-00249348}

Submitted on 1 Jan 1995

HAL is a multi-disciplinary open access archive for the deposit and dissemination of scientific research documents, whether they are published or not. The documents may come from teaching and research institutions in France or abroad, or from public or private research centers.
L'archive ouverte pluridisciplinaire HAL, est destinée au dépôt et à la diffusion de documents scientifiques de niveau recherche, publiés ou non, émanant des établissements d'enseignement et de recherche français ou étrangers, des laboratoires publics ou privés. 
Classification

Physics Abstracts

$06.70 \mathrm{M}$

\title{
Nouvelle structure de capteur à courants de Foucault et algorithme associé pour la mesure des propriétés électrique et magnétique d'un métal
}

\author{
Minh-Quang Lê et Dominique Placko \\ Laboratoire d'Electricité-Signaux- Robotique, Unité associée au C.N.R.S, D1375, 61 avenue du \\ Président Wilson, 94235 Cachan Cedex, France
}

(Reçu le 4 août 1994, révisé 12 janvier 1995, accepté le 20 mars 1995)

\begin{abstract}
Résumé. - Cet article décrit une nouvelle structure de capteur à courants de Foucault qui permet d'améliorer la sensibilité du capteur tout en conservant sa résolution latérale. Nous avons développé un modèle analytique de ce capteur qui peut être inversé pour estimer la conductivité électrique, la perméabilité magnétique d'une cible homogène et/ou la distance capteur-cible. La procédure d'inversion a été décrite et montre une possibilité d'estimer ces trois paramètres avec précision. L'expérience a été effectuée sur des métaux magnétiques et amagnétiques.
\end{abstract}

\begin{abstract}
This paper describes a new structure for eddy-current transducers which improves sensitivity while maintaining high lateral resolution. We have developed an analytical model to allow a model-based inversion from transducers signals to estimate thick and homogeneous plates electrical conductivity, magnetic permeability and/or lift-off. The inversion procedure is described and shows the possibility of a precise simultaneous estimation of the three parameters. Experiments were conducted with magnetic and non magnetic metals.
\end{abstract}

\section{Introduction}

Les capteurs à courants de Foucault (CCF) sont utilisés depuis plus d'un siècle pour le contrôle des pièces conductrices notamment métalliques. De nos jours, la théorie des capteurs à courants de Foucault est déjà largement développée. Ils trouvent leurs applications dans divers domaines industriels qui vont de la mesure des propriétés de la matière, des mesures dimensionnelles jusqu'à la détection de défauts dans les pièces mécaniques.

Le CCF élémentaire se compose simplement d'un bobinage à air muni de $N$ spires parcourues par un courant alternatif $i_{\text {exc }}$. Pour caractériser le milieu sondé par le CCF, on peut introduire la notion de réluctance ou de perméance complexe si on exprime le rapport entre la différence de potentiels magnétiques $\Delta$ et le flux $\Phi$ émis par le CCF :

$$
\mathrm{R}=\frac{\Delta}{\Phi}=\frac{1}{\mathrm{P}}
$$


$\Delta$ étant fixée par les ampères-tours d'excitation : $\Delta=N i_{\text {exc }}$. On retrouve alors la relation entre l'impédance complexe $Z$ du CCF et la perméance $P$ :

$$
Z=\frac{V}{i_{\text {exc }}}=\frac{j \omega N^{2}}{\mathrm{R}}=j \omega N^{2} \mathrm{P}=R+j X
$$

En pratique, même en l'absence de la cible, $Z$ possède une composante résistive $R_{0}$. Pour s'affranchir de $R_{0}$ et de la croissance des termes avec la fréquence, on préfère souvent travailler avec l'impédance normalisée $Z_{\mathrm{n}}$.

$$
Z_{\mathrm{n}}=\frac{R-R_{0}}{X_{0}}+j \frac{X}{X_{0}}=R_{\mathrm{n}}+j X_{\mathrm{n}}
$$

où $X_{0}$ est la composante réactive (partie imaginaire) de $Z$ en l'absence de la cible. $Z_{\mathrm{n}}$ varie en fonction des propriétés de la cible, de la distance capteur-cible, de la fréquence du courant d'excitation et des dimensions du capteur. Dans le cas d'une cible homogène et pour un capteur donné, les paramètres influents sont la fréquence du courant d'excitation du capteur $f$, la conductivité électrique $\sigma$, la perméabilité magnétique $\mu$ et la distance capteur-cible $d$.

Pour augmenter l'efficacité de détection du CCF, on le pourvoit généralement d'un circuit magnétique sur lequel sont disposés un ou plusieurs bobinages. Le circuit magnétique permet de focaliser l'énergie émise par le circuit d'excitation vers le milieu analysé. On peut classer succinctement les capteurs en deux catégories : les structures à symétrie de révolution (généralement un "pot" en ferrite, Fig. 1c) et les structures sans symétrie de révolution comme les structures en U (Fig. 1b).

De nombreuses études ont été effectuées dans le monde sur la modélisation analytique des structures des capteurs à courants de Foucault. On peut citer simplement les références [3-5, $7,16]$ pour les CCF sans circuit magnétique et les références $[8,9,11]$ pour les CCF avec circuit magnétique de forme $\mathrm{U}$.

La modélisation des structures avec circuit magnétique de forme U est effectivement difficile à cause du nombre d'interfaces entre les différents milieux qui le composent. Pour les capteurs de forme U entourés par un blindage électromagnétique (Fig. 1e), la modélisation peut s'effectuer par le biais de la méthode des images électriques où les pôles du capteur sont assimilés à des charges ponctuelles [7-9,11] (Fig. 1f). Cette approche est particulièrement intéressante, on peut en résumer le principe comme suit : les charges magnétiques ponctuelles qui représentent les pôles du capteur se trouvent devant deux plans métalliques, celui de la cible et celui du blindage lié au capteur lui-même. Les deux plans sont considérés comme infiniment étendus. Leurs effets sont alors facilement modélisables grâce à la théorie des images électriques. Les réflexions successives des champs magnétiques sur le blindage et sur le plan conducteur de la cible font apparaître une double infinité de dipôles magnétiques (Fig. 1f). Le calcul de la réluctance conduit, par conséquent, à l'expression d'une série mathématique infinie qui est aisément exploitable.

Certains auteurs ont essayé de modéliser le capteur de forme "pot" mais leurs modèles sont plutôt complexes et difficilement exploitables [4]. Par ailleurs, ce type de capteur présente des fuites magnétiques importantes et un rapport portée/encombrement moins favorable que les capteurs $\mathrm{U}$.

Quand le modèle analytique associé à un CCF est trop complexe, donc coûteux en temps de calcul, ou trop imprécis, on préfère modéliser ce CCF expérimentalement. A partir des mesures sur des cibles "connues", on essaye de décrire la relation "entrées-sorties" du CCF par une expression mathématique adéquate. Cette étape de "calibration" ou d' "étalonnage" est parfois impossible à réaliser, faute d'échantillons de propriétés connues. 

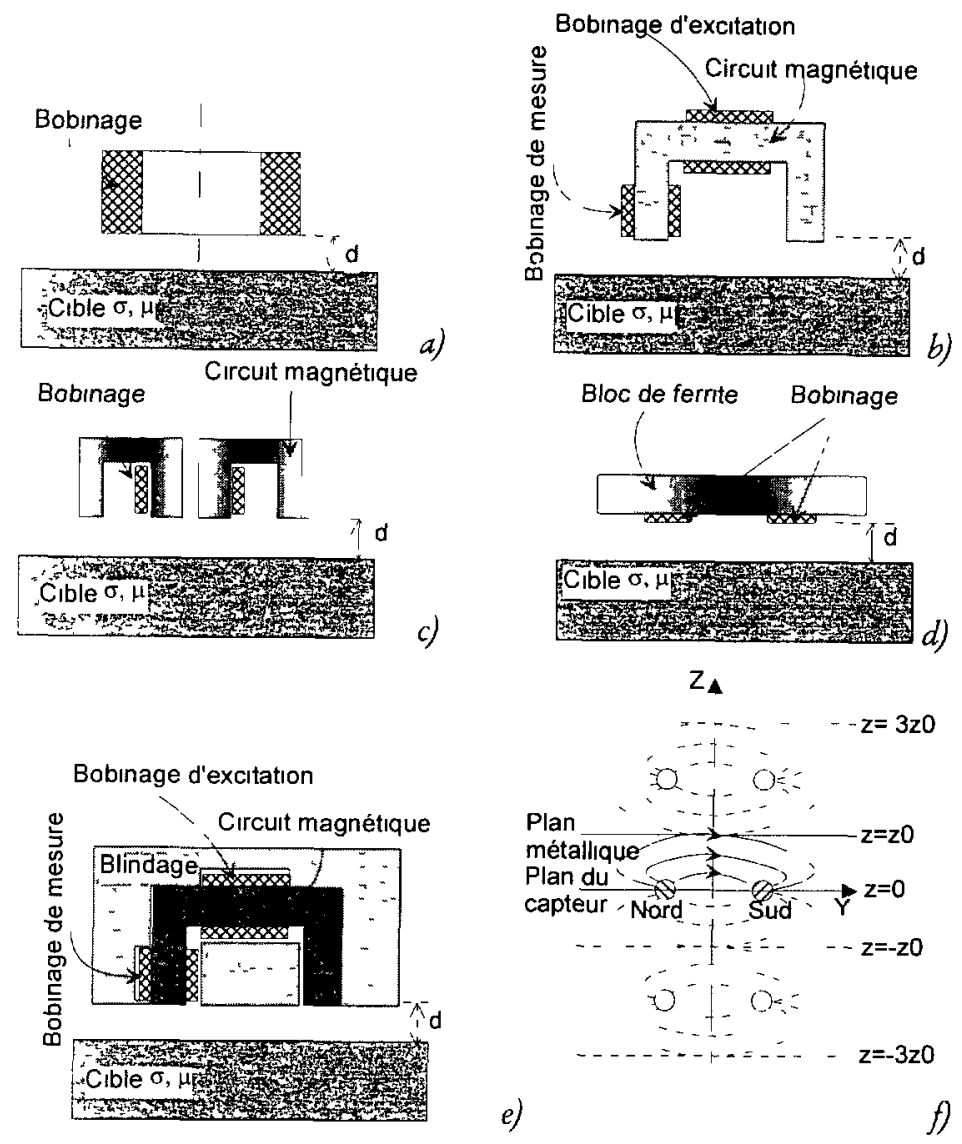

Fig. 1. - Les formes usuelles de capteurs à courants de Foucault : a) CCF élémentaire, b) CCF de forme "U", c) CCF de forme "pot". d) Structure proposée, e) CCF blindé, f) Méthode des images électriques.

[Usual structures of eddy-current sensors. a) Elementary sensor, b) U-core sensor, c) Cup-core sensor d) Proposed structure, e) Shielded sensor, f) Electrical images method.]

La structure que nous proposons ici se prête bien à une modélisation analytique exploitable. De plus, elle présente une bonne sensibilité vis à vis des propriétés de la cible tout en gardant un faible encombrement (Fig. 1d), ce qui permet d'augmenter la résolution spatiale du capteur.

\section{Structure proposee et modele analytique associe}

2.1. InTRODUCTION. - Le CCF à bobinage sans noyau est facile à modéliser mais il n'est pas très sensible car la distribution dans l'espace de l'énergie émise par le capteur n'est pas optimale. On peut augmenter la sensibilité du CCF en augmentant son diamètre, mais ceci nuit à sa résolution latérale.

Examinons un CCF à bobinage sans noyau de longueur $l$, rayon interne $r_{1}$ et rayon externe $r_{2}$ (Fig. 2a). Pour augmenter la sensibilité du CCF, nous savons qu'il faut augmenter l'intensité du champ magnétique $\mathbf{H}$ dans les régions 3 et 4 et la diminuer dans les régions 1,2 et 2-3 [10]. Considérons le champ magnétique le long d'un circuit L (Fig. 2a.). Selon le théorème d'Ampère, 

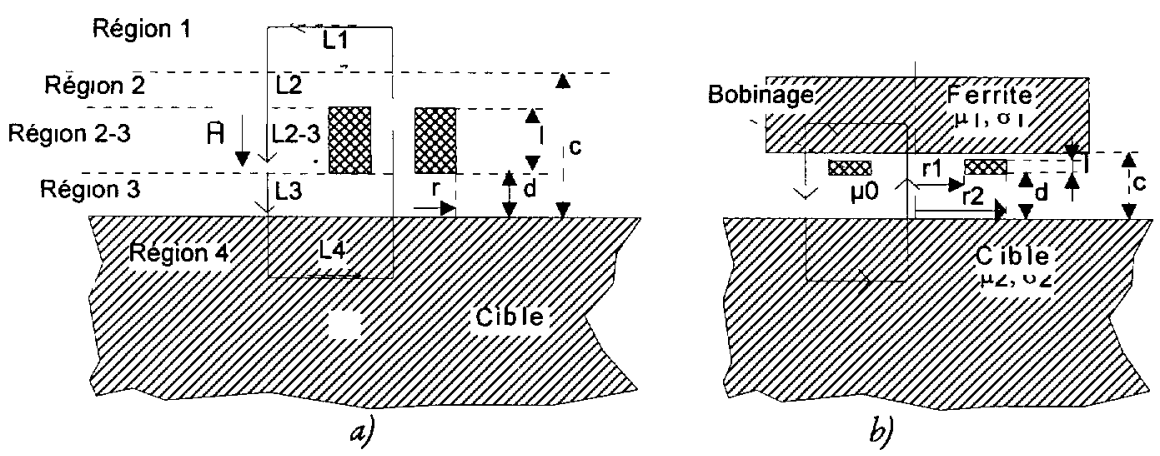

Fig. 2. - a) Analyse de la distribution du champ magnétique, b) Structure de CCF proposée. [a) Analysis of magnetic field distribution, b) Structure of proposed sensor.]

on a :

$$
N i_{\mathrm{exc}}=\int_{\mathrm{L} 1} \mathbf{H} \cdot \mathrm{d} \mathbf{l}+2 \int_{\mathrm{L} 2} \mathbf{H} \cdot \mathrm{d} \mathbf{l}+2 \int_{\mathrm{L} 2-3} \mathbf{H} \cdot \mathrm{d} \mathbf{l}+2 \int_{\mathrm{L} 3} \mathbf{H} \cdot \mathrm{d} \mathbf{l}+\int_{\mathrm{L} 4} \mathbf{H} \cdot \mathrm{d} \mathbf{l}
$$

où $N i_{\text {exc }}$ exprime les ampères-tours d'excitation.

Il faut donc minimiser les quantités $Q_{1}=\int_{\mathrm{L} 1} \mathbf{H} \cdot \mathrm{dl}, Q_{2}=2 \int_{\mathrm{L} 2} \mathbf{H} \cdot \mathrm{d} \mathbf{l}$ et $Q_{3}=2 \int_{\mathrm{L} 2-3} \mathbf{H} \cdot \mathrm{d} \mathbf{l}$ Pour minimiser $Q_{1}$, on peut diminuer l'intensité de $\mathbf{H}$ dans la région 1 en disposant dans cette région un matériau de grande perméabilité magnétique (ferrite) (ce plan joue le rôle d'un plan "réflecteur" au sens des images électriques [11]). Quant à $Q_{2}$ et $Q_{3}$, il faut diminuer les parcours de $\mathbf{H}$ dans l'air L2 et L2-3. Pour ce faire, le bobinage doit être collé de pluss près possible du plan de ferrite pour diminuer L2 et il doit aussi être très plat pour que L2-3 soit faible (la longueur $l$ de la bobine est faible devant son rayon moyen $r$ ).

Pour vérifier toutes ces considérations, nous avons effectué des simulations à l'aide d'un logiciel de calcul de champs électromagnétiques par la méthode des éléments finis, Maxwell ${ }^{(R)}$, développé par Ansoft Company. La figure 3a présente une comparaison des sensibilités par rapport aux variations de la distance capteur-cible $d$ entre le capteur proposé ici et un capteur classique de type pot de même encombrement. La figure $3 b$ présente l'évolution de la sensibilité du capteur proposé par rapport à la conductivité de la cible en fonction de ses dimensions. On peut remarquer que pour les mêmes variations de la distance capteur-cible, les courbes $1,2,3$ et 4 sont plus espacées que les courbes $5,6,7$ et 8 , ce qui signifie que le nouveau capteur est plus sensible que le capteur de type pot (Fig. 3a.). D'autre part, plus le capteur est "plat", plus il est sensible à la conductivité (Fig. 3b.).

2.2. Modélisation. - Dodd et al. ont proposé plusieurs modèles de CCF à bobinage à air dans une série d'articles [7]. La relation $Z=\Theta\left(f, \mu, \sigma, \mathrm{d}, r_{1}, r_{2}, l\right)$ a été élaborée sous forme analytique. $\Theta$ est une fonction complexe dans laquelle interviennent les fonctions de Bessel. Ces modèles correspondent à des configurations diverses et montrent une bonne adéquation avec les données expérimentales.

Pour obtenir le modèle analytique correspondant à notre structure de capteur, nous avons utilisé une méthode analogue à celle de Dodd et al. [7]. A partir de la 2ème équation de Maxwell $\nabla \times \mathbf{H}=\mathbf{J}$ et en utilisant les définitions $\mathbf{J}=\sigma \mathbf{E}, \mathbf{B}=\mu \mathbf{H}, \nabla \times \mathbf{A}=\mathbf{B}$ nous avons : 


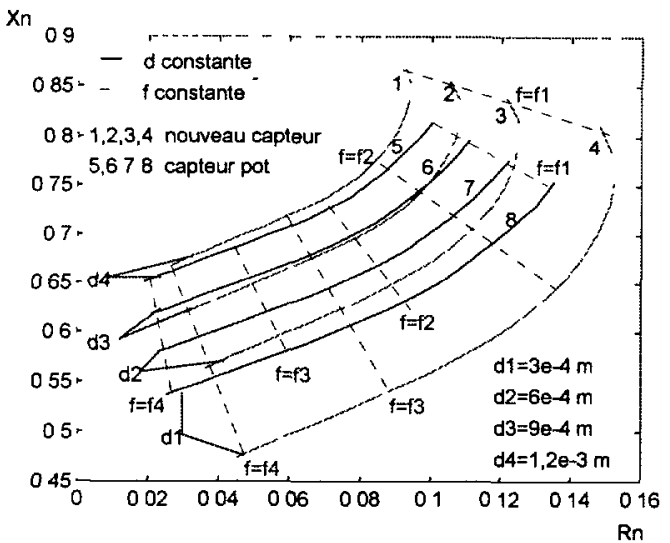

a)

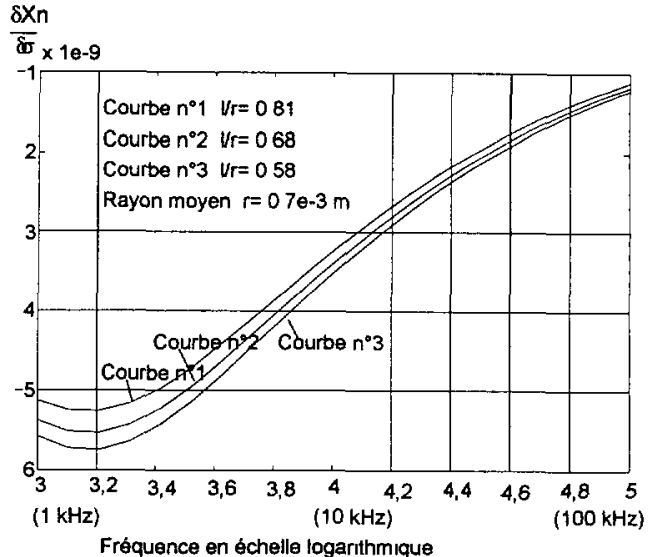

b)

Fig. 3. - a) Comparaison de performances entre le nouveau capteur et un capteur "pot". b) Evolution de la sensibilité du capteur en fonction du rapport $l / r$.

[a) Performances comparison between the proposed sensor and a classical cup-core sensor. b) Sensor sensitivity as a function of ratio $l / r$.]

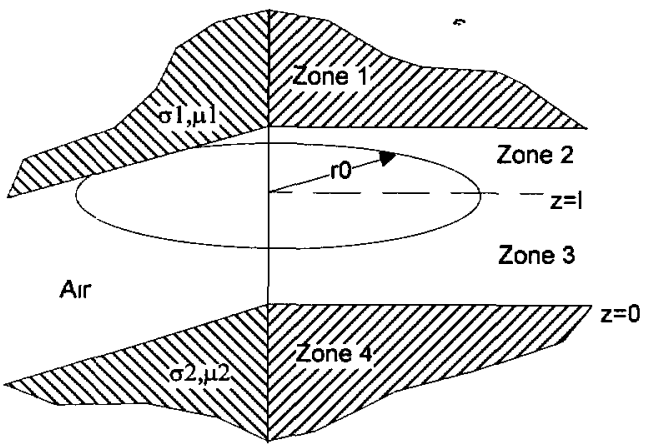

a)

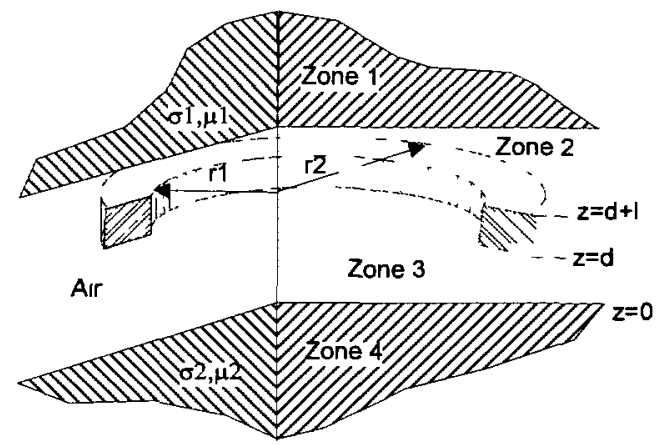

b)

Fig. 4. - a) Géométrie avec une spire élémentaire, b) Géométrie réelle.

[a) Delta-function coil on the examined geometry. b) Real coil on the examined geometry.]

$$
\nabla \times\left(\frac{1}{\mu} \nabla \times \mathbf{A}\right)=-\sigma\left(\frac{\partial \mathbf{A}}{\partial t}+\nabla \boldsymbol{\Phi}\right)=\mathbf{J}_{\mathrm{e}}+\mathbf{J}_{s}
$$

où $\mathbf{A}$ est le potentiel vecteur magnétique, $\mathbf{J}_{\mathrm{e}}=-\sigma \frac{\partial \mathbf{A}}{\partial t}$ la densité des courants de Foucault et $\mathbf{J}_{\mathrm{s}}=-\sigma \nabla \Phi$ la densité du courant d'excitation ( $\nabla \Phi$ représente un champ électrique). En considérant un milieu linéaire et de perméabilité constante [10], l'équation (3) devient :

$$
\nabla(\nabla \mathbf{A})-\nabla^{2} \mathbf{A}=-\mu \sigma \frac{\partial \mathbf{A}}{\partial t}-\mu \mathbf{J}_{\mathbf{s}}
$$

Considérons le problème en coordonnées cylindriques $r, z, \theta$. Puisqu'il est complètement axisymétrique, seules les composantes $\mathrm{A}_{\theta}$ et $\mathrm{J}_{\mathbf{s} \theta}$ existent. Si le courant d'excitation est sinusoïdal, on a $\frac{\partial \mathbf{A}}{\partial t}=j \omega \mathbf{A}$. En choisissant une condition de jauge convenable pour que $\nabla \mathbf{A}$ soit nul, 
l'équation (4) devient :

$$
\frac{\partial^{2} \mathrm{~A}}{\partial \mathrm{r}^{2}}+\frac{1}{\mathrm{r}} \frac{\partial \mathrm{A}}{\partial \mathrm{r}}+\frac{\partial^{2} \mathrm{~A}}{\partial \mathrm{z}^{2}}-\frac{\mathrm{A}}{\mathrm{r}^{2}}=\mu \mathrm{J}_{\mathrm{s}}+\mathrm{j} \omega \mu \sigma \mathrm{A}
$$

en coordonnées cylindriques. Dans l'équation (5), A et $\mathrm{J}_{\mathbf{s}}$ signifient $\mathrm{A}_{\theta}$ et $\mathrm{J}_{\mathbf{s} \theta}$. Dans les régions qui ne sont pas parcourues par $\mathrm{J}_{\mathbf{s}}$, la solution générale de l'équation (5) est de la forme

$$
\mathrm{A}(r, z)=\int_{0}^{\infty}\left[A \mathrm{e}^{\alpha_{\jmath} z}+B \mathrm{e}^{-\alpha_{1} z}\right]\left[C J_{1}(\alpha r)+D Y_{1}(\alpha r)\right] \mathrm{d} \alpha
$$

où $\mathrm{A}(r, z)$ signifie que $\mathrm{A}$ est une fonction de $r$ et de $z, J_{1}(x)$ et $Y_{1}(x)$ sont des fonctions de Bessel, $\alpha$ est la constante de séparation utilisée pour la résolution de l'équation (5). $A, B, C$, $D$ sont les fonctions de $f, \mu, \sigma, d, r_{1}, r_{2}$, et $l$. Elles seront déterminées à partir des conditions aux limites entre les différentes régions.

Les conditions aux limites entre deux régions successives $i$ et $i+1$ sont

$$
\begin{gathered}
\mathrm{A}^{(\mathrm{i})}=\mathrm{A}^{(\mathrm{i}+1)} \\
\frac{1}{\mu^{(\mathrm{i})}} \frac{\partial \mathrm{A}^{(\mathrm{i})}}{\partial z}=\frac{1}{\mu^{(\mathrm{i}+1)}} \frac{\partial \mathrm{A}^{(\mathrm{i}+1)}}{\partial z}-\mu_{0} \mathrm{~J}_{\mathrm{s}}(r, z)
\end{gathered}
$$

Dans l'équation (8), $\mathrm{J}_{\mathrm{s}}(r, z)$ signifie que la densité de courant est une fonction $r$ de et de $z$.

Nous trouvons ainsi les expressions du potentiel vecteur magnétique $\mathrm{A}(r, z)$ engendré par une densité de courant $J_{\mathbf{s}}(r, z)$ dans les différentes régions. Pour calculer l'expression de $\mathrm{A}(r, z)_{\text {total }}$ créé par la bobine réelle, nous appliquons la formule :

$$
A(r, z)_{\text {total }}=\int_{r_{1}}^{\mathrm{r}_{2}} \int_{\mathrm{l}_{1}}^{\mathrm{l}_{2}} A\left(r, z, r^{\prime}, z^{\prime}\right) \mathrm{d} r^{\prime} \mathrm{d} z^{\prime}
$$

où $\mathrm{A}\left(r, z, r^{\prime}, z^{\prime}\right)$ est le potentiel vecteur magnétique créé à $(r, z)$ par une densité de courant $\mathrm{J}_{\mathbf{s}}\left(r^{\prime}, z^{\prime}\right)$, qui peut être déduite du courant d'excitation $i_{\text {exc }}$.

Une fois l'expression $\mathrm{A}(r, z)_{\text {total }}$ obtenue, on peut accéder facilement à la tension induite dans la bobine. Sur une spire $i$, la tension induite $V_{\imath}$ est

$$
V_{\imath}=j \omega \int_{L} \mathrm{~A}\left(r_{\mathrm{i}}, z_{\mathrm{i}}\right)_{\text {total }} \mathrm{d} L
$$

$L$ étant la longueur de la spire. Puisque chaque spire est considérée comme axisymétrique et de rayon $r_{\imath}, V_{\imath}$ devient

$$
V_{\imath}=j \omega 2 \pi r_{2} \mathrm{~A}\left(r_{i}, z_{i}\right)
$$

La tension induite totale dans la bobine de $N$ spires est

$$
V_{\text {totale }}=j \omega 2 \pi \sum_{\mathrm{i}}^{N} r_{\imath} \mathrm{A}\left(r_{\imath}, z_{\imath}\right)
$$

$N$ est le nombre de spires du bobinage d'excitation, $i=1 . . N$.

L'impédance de la bobine est

$$
Z=\frac{V_{\text {totale }}}{i_{\text {exc }}}=\Theta\left(f, \mu, \sigma, d, r_{1}, r_{2}, l\right)
$$

Le lecteur peut trouver l'expression complète de $Z$ en annexe 
2.3. VAlidation DU MODÈle. - La formule (13) a été codée en langage Turbo Pascal 5.5. Les résultats obtenus sont en très bonne adéquation avec les mesures expérimentales (Fig. 5).

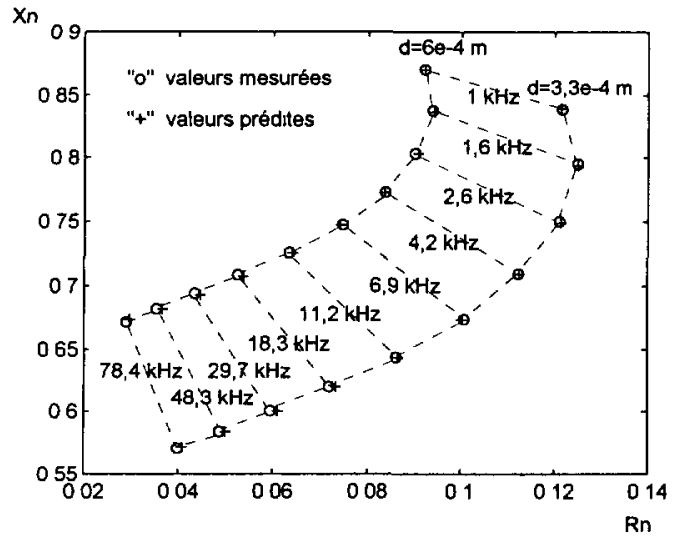

a)

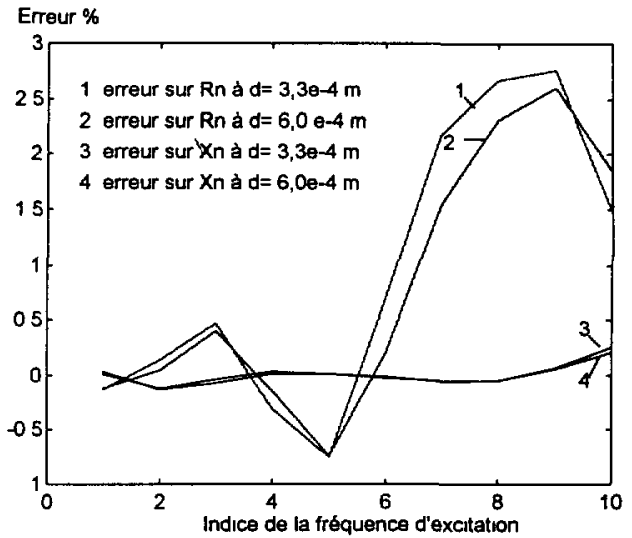

b)

Fig. 5. - a) Valeurs d'impédance normalisée mesurées et prédites par le modèle. b) Ecarts relatifs en pourcentage entre les valeurs mesurées et les valeurs prédites.

(a) Measured and predicted values of normalized impedance. b) Relative error between measured and predicted values.]

L'expérience a été effectuée sur une cible plane et épaisse en laiton ( $\left.\mu_{\mathrm{r}}=1, \sigma=14 \mathrm{MS} / \mathrm{m}\right)$ pour deux distances capteur-cible différentes. Les mesures sont effectuées à 10 fréquences d'excitation du capteur. Les valeurs de $R_{\mathrm{n}}$ et de $X_{\mathrm{n}}$ sont portées sur un plan appelé "plan d'impédance normalisée" (Fig. 5a).

\section{Probleme inverse : recherche des parametres $\mu_{\mathrm{r}}, \sigma$ et $d$ d'une cible inconnue.}

3.1. GÉNÉRALITÉs. - Les capteurs à courants de Foucault sont utilisés pour contrôler les pièces métalliques. Les paramètres $\mu_{\mathrm{r}}$ et $\sigma$ d'un objet métallique dépendent fortement des contraintes mécaniques et thermiques que le matériau a subi et permettent donc d'apprécier sa qualité de fabrication (à partir de ce paragraphe, nous traitons la perméabilité relative $\mu_{\mathrm{r}}$ au lieu de la perméabilité absolue $\mu$ ). Par ailleurs, la distance entre le capteur et la pièce contrôlée constitue l'un des paramètres les plus influents sur les données brutes fournies par le capteur. Sachant qu'elle ne peut être maintenue constante pour des raisons évidentes, ou bien que l'accès à ce paramètre présente un intérêt particulier (contrôle dimensionnel de pièces métalliques), nous allons considérer le cas général où l'ensemble des trois paramètres $\mu_{\mathrm{r}}, \sigma$ et $d$ d'une cible plane doivent être déterminés simultanément. Le capteur utilisé est celui de la figure 1d. La cible est supposée très épaisse par rapport à la profondeur de pénétration du champ magnétique pour que son épaisseur n'intervienne pas dans les résultats de mesures. Le problème inverse consiste à estimer les paramètres en question $\left(\mu_{\mathbf{r}}, \sigma, d\right)$ à partir de la connaissance des signaux capteur. Ces signaux peuvent être obtenus de plusieurs façons. On peut exciter le capteur par un courant sinusoïdal de fréquence variable et effectuer une mesure à chaque fréquence (méthode multifréquences). Le capteur peut aussi être excité par une impulsion de courant et les mesures sont faites à différents instants durant la réponse impulsionnelle (méthode impulsionnelle). En 


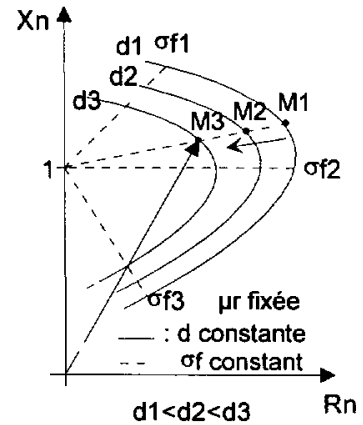

a)

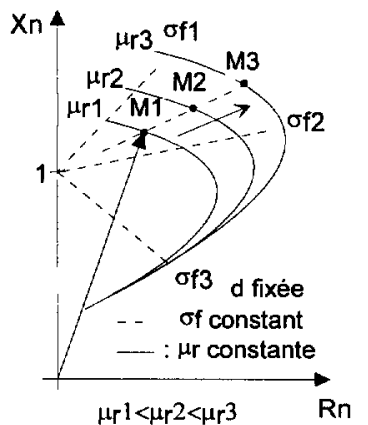

b)

Fig. 6. - Influences des paramètres sur le plan d'impédance. a) Influence de $d$, b) Influence de $\mu_{\mathrm{r}}$. [Influences of parameters on normalized impedance plane. a) Influence of $d$. b) Influence of $\mu_{\mathrm{r}}$.]

général, le nombre d'informations disponibles sur la cible doit être supérieur ou égal au nombre de paramètres inconnus. Nous allons discuter seulement de la méthode multi-fréquences.

Dans cette méthode, à chaque fréquence, deux informations peuvent être obtenues : la partie réelle $R_{\mathrm{n}}$ et la partie imaginaire $X_{\mathrm{n}}$ de l'impédance normalisée $Z_{\mathrm{n}}$. Par conséquent, si $M$ fréquences sont utilisées, $2 M$ informations sont disponibles. De plus, ces fréquences doivent être choisies de telle sorte qu'elles donnent des informations complémentaires sur les paramètres inconnus. La figure 6 présente les différents effets liés aux variations de $\mu_{\mathrm{r}}, \sigma$ ou $d$ sur le point de fonctionnement $M$ dans le plan d'impédance normalisée.

Sur la figure 6 , on peut constater que les influences de $\mu_{\mathrm{r}}, \sigma$ et $d$ sont différentes selon les valeurs prises par le produit $\sigma f$. Ce point suggère qu'il faille choisir les fréquences du courant d'excitation telles que le point de fonctionnement $M$ dans le plan d'impédance normalisée couvre effectivement les trois domaines distincts repérés sur la figure 6 par $\sigma f_{1}$, $\sigma f_{2}$ et $\sigma f_{3}$ pour avoir des informations suffisamment riches et complémentaires sur $\mu_{\mathrm{r}} \sigma$, et $d$. A titre d'exemple, pour contrôler un acier dont $\sigma=1 \mathrm{MS} / \mathrm{m}$, nous avons choisi 3 fréquences, $1 \mathrm{kHz}$, $50 \mathrm{kHz}, 100 \mathrm{kHz}$, donnant respectivement un produit $\sigma f$ de $1 \times 10^{9} \mathrm{HzS} / \mathrm{m}, 50 \times 10^{9} \mathrm{HzS} / \mathrm{m}$, $100 \times 10^{9} \mathrm{HzS} / \mathrm{m}$.

3.2. Modèle APPROChÉ AUTOUR D'Un POINT DE FONCTIONNEMENT. - Lorsque les paramètres recherchés ne varient que faiblement autour d'un point de fonctionnement $\mathrm{P}_{0}\left(d_{0}, \mu_{r_{0}}, \sigma_{0}\right)$ (cas souvent rencontré dans le contrôle de qualité des pièces) et provoquent une variation quasilinéaire des sorties du CCF $R_{\mathrm{n}}$ et $X_{\mathrm{n}}$, on peut établir directement la relation inverse suivante

$$
\boldsymbol{\theta}^{\mathrm{t}}=\gamma^{\mathrm{t}} \times \mathbf{C}
$$

où $\boldsymbol{\theta}=\left[\mu_{\mathrm{r}}, \sigma, \mathrm{d}\right]^{\mathrm{t}}$ est le vecteur des paramètres inconnus

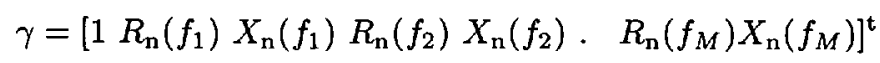

$M$ est le nombre de fréquences d'excitation.

$R_{\mathrm{n}}\left(f_{1}\right) X_{\mathrm{n}}\left(f_{1}\right) R_{\mathrm{n}}\left(f_{2}\right) X_{\mathrm{n}}\left(f_{2}\right)$. $R_{\mathrm{n}}\left(f_{M}\right), X_{\mathrm{n}}\left(f_{M}\right)$ sont les sorties du CCF obtenues aux fréquences d'excitation $f_{1}, f_{2}, . ., f_{M}$.

C est la matrice des coefficients du modèle linéaire 


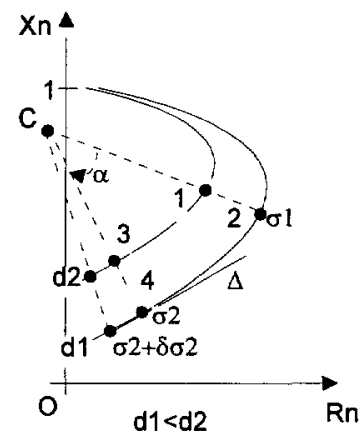

Fig. 7. - Changement de variables en vue d'améliorer la linéarité du modèle approché. [Change of variables for improving the linearity of the approximated model.]

$$
\mathbf{C}=\left[\begin{array}{lll}
c_{11} & c_{12} & c_{13} \\
c_{21} & c_{22} & c_{23} \\
\cdot & \ddot{v} & \ddot{c_{\imath 3}} \\
c_{\imath 1} & c_{\imath 2} & c_{\imath 3} \\
\cdot & & \\
c_{2 M+1,1} & c_{2 M+1,2} & c_{2 M+1,3}
\end{array}\right]
$$

La détermination de $\mathbf{C}$ s'effectue au cours de l'étape d'étalonnage. Cela consiste à présenter au système de mesure $Q$ différents échantillons dont les propriétés $\mu_{\mathrm{r} \imath}, \sigma_{\imath}, d_{2}$ sont connues et à enregistrer les sorties $R_{\mathrm{n} \imath}, X_{\mathrm{n} \imath}, i=1$.. $Q$ avec $Q \geq 2 M+1$. Avec un modèle analytique précis, on peut aussi calculer ces valeurs de sorties au lieu de faire des mesures. $\mathbf{C}$ est calculée par la méthode des moindres carrés

$$
\mathbf{C}=\left(\Psi^{\mathrm{t}} \times \Psi\right)^{-1} \times \Psi^{\mathrm{t}} \times \mathbf{P}_{\text {cal }}
$$

où $\Psi$ s'appelle "la matrice du plan d'expérience". Chaque ligne de $\Psi$ est un vecteur de sorties $\gamma^{\mathrm{t}}$ obtenu pour un échantillon précis. La taille de $\Psi$ est $Q \times(2 M+1) . \mathbf{P}_{\text {cal }}$ est la matrice des valeurs d'étalonnage. Chaque ligne de $\mathbf{P}_{\text {cal }}$ est $\mathbf{P}_{\imath}=\theta_{\imath}=\left[\begin{array}{lll}\mu_{\mathrm{r} 2} & \sigma_{\imath} & d_{\imath}\end{array}\right]^{t}, \mu_{\mathrm{r} \imath}, d_{\imath}$ étant les valeurs de calibration.

Les valeurs des éléments de $\mathbf{C}$ seront utilisées dans le modèle (14) pour déterminer les paramètres des échantillons inconnus.

Lorsque $R_{\mathrm{n}}$ et $X_{\mathrm{n}}$ ne varient plus linéairement en fonction des paramètres influents, on peut toujours essayer d'utiliser un changement de variable pour "linéariser" la relation "entréessorties" du capteur. Par exemple, quand la conductivité connaît de fortes variations, l'extrémité du vecteur d'impédance normalisée se déplace sur une courbe dont l'allure suggère qu'un angle devrait mieux représenter l'évolution de la conductivité que $R_{\mathrm{n}}$ et $X_{\mathrm{r}}$. De plus, il doit être invariant par rapport aux variations de la distance capteur-cible $d$. Cet angle ne peut donc être que l'angle entre les deux droites, qui passent respectivement par les points 1,2 et 3,4 (Fig. 7). Cette méthode permet d'étendre la validité du modèle local linéaire à des variations plus grandes de la conductivité. Une approche analogue avait été développée dans la référence [7]. Notons que cette approche est rarement applicable à l'ensemble du domaine de variation des paramètres étudiés.

Il est aussi possible de travailler avec $\ln \left(R_{n}\right)$ et $\ln \left(X_{n}\right)$ plutôt qu'avec $R_{n}$ et $X_{n}$ afin d'obtenir une relation quasi-linéaire. Cette idée est également justifiée par la forme du modèle (13) (voir 
aussi l'annexe), qui comprend des termes exponentiels. Cette méthode a été utilisée dans la référence [8].

3.3. INVERSION GÉnÉRALE ET ALGORITHME ITÉRATIF. - Lorsqu'on ne dispose pas d'un nombre suffisant d'échantillons de propriétés connues pour faire la calibration, il faut avoir recours à un modèle de connaissance du type (13) et l'inverser. Cela consiste à trouver la combinaison des paramètres inconnus $d, \sigma$ et $\mu_{\mathrm{r}}$ qui satisfait le système d'équation non-linéaire :

$$
\begin{aligned}
& R_{\mathrm{n}}\left(d, \sigma, \mu_{\mathrm{r}}, f_{1}\right)=R_{\mathrm{n}_{1}} \\
& X_{\mathrm{n}}\left(d, \sigma, \mu_{\mathrm{r}}, f_{1}\right)=X_{\mathrm{n}_{1}} \\
& \ldots \\
& R_{\mathrm{n}}\left(d, \sigma, \mu_{\mathrm{r}}, f_{M}\right)=R_{\mathrm{n}_{M}} \\
& X_{\mathrm{n}}\left(d, \sigma, \mu_{\mathrm{r}}, f_{M}\right)=X_{\mathrm{n}_{M}}
\end{aligned}
$$

où $M$ est toujours le nombre de fréquences d'excitation, et $R_{\mathrm{n}_{1}}, X_{\mathrm{n}_{1}}, \ldots, R_{\mathrm{n}_{\mathrm{M}}}, X_{\mathrm{n}_{\mathrm{M}}}$ sont les valeurs mesurées de $R_{\mathrm{n}}$ et de $X_{\mathrm{n}}$ à $M$ fréquences différentes. Le nómbre d'équations dans le système (17a) est $2 M$. Sous forme vectorielle, (17a) s'écrit :

$$
\mathbf{F}(\theta)=\eta
$$

où $\theta=\left[\begin{array}{lll}d & \sigma & \mu_{\mathrm{r}}\end{array}\right]^{\mathrm{t}}, \eta=\left[\begin{array}{lll}R_{1} & X_{1} & R_{M} X_{M}\end{array}\right]^{\mathrm{t}}$

et $\mathbf{F}(\theta)=\left[\begin{array}{llll}R_{\mathrm{n}}\left(\theta, f_{1}\right) & X_{\mathrm{n}}\left(\theta, f_{1}\right) & R_{\mathrm{n}}\left(\theta, f_{M}\right) & X_{\mathrm{n}}\left(\theta, f_{M}\right)\end{array}\right]^{\mathrm{t}}$

Quand le nombre d'équations est supérieur au nombre de paramètres inconnus (cas très souvent rencontré en pratique), ce système d'équations non-linéaire devient surdéterminé et nous allons le résoudre de façon approchée par la méthode des moindres carrés. La solution est une combinaison de $d, \sigma$ et $\mu_{\mathrm{r}}$ qui minimise l'erreur quadratique

$$
\begin{aligned}
\varepsilon & =\left\{R_{\mathrm{n}}\left(d, \sigma, \mu_{\mathrm{r}}, f_{1}\right)-R_{\mathrm{n}_{1}}\right\}^{2}+\left\{X_{\mathrm{n}}\left(d, \sigma, \mu_{\mathrm{r}}, f_{1}\right)-X_{\mathrm{n}_{1}}\right\}^{2}+ \\
& +\left\{R_{\mathrm{n}}\left(d, \sigma, \mu_{\mathrm{r}}, f_{M}\right)-R_{\mathbf{n}_{M}}\right\}^{2}+\left\{X_{\mathrm{n}}\left(d, \sigma, \mu_{\mathrm{r}}, f_{M}\right)-X_{\mathbf{n}_{M}}\right\}^{2}
\end{aligned}
$$

Ce problème peut être résolu par l'algorithme itératif de Gauss-Newton. Nous allons proposer en outre une méthode qui permet de coupler le modèle approché (14) avec l'algorithme de Gauss-Newton.

3.3.1. Algorithme de Gauss-Newton. - Cette méthode consiste à linéariser à chaque itération chacune des équations de (17a) autour d'un point de fonctionnement correspondant à la solution approchée obtenue à l'itération précédente (ceci implique une initialisation effectuée par l'utilisateur de l'algorithme). On obtient ainsi un système d'équations linéaires surdéterminé sous la forme vectorielle suivante :

$$
\mathbf{F}(\theta) \equiv \mathbf{F}\left(\theta^{(2)}\right)+\nabla \mathbf{F}\left(\theta^{(2)}\right)\left(\theta-\theta^{(\imath)}\right)=\eta
$$

où $\nabla \mathbf{F}\left(\theta^{(\imath)}\right)$ est la matrice jacobienne de la fonction vectorielle à $\mathbf{F}(\theta)$ à $\theta=\theta^{(2)}$

La solution de (19) au sens des moindres carrés est :

$$
\theta=\theta^{(2)}+\left[\nabla \mathbf{F}\left(\theta^{(2)}\right)^{\mathrm{t}} \nabla \mathbf{F}\left(\theta^{(2)}\right)\right]^{-1} \nabla \mathbf{F}\left(\theta^{(\imath)}\right)^{\mathrm{t}}\left[\eta-\mathbf{F}\left(\theta^{(2)}\right)\right]
$$

Cette solution n'est pas forcément la meilleure car l'approximation linéaire pourrait être grossière. On reporte donc cette solution dans le système (17a) et on le linéarise autour du point de fonctionnement donné par la nouvelle solution, d'où la forme itérative suivante 


$$
\theta^{(\imath+1)}=\theta^{(2)}+\left[\nabla \mathbf{F}\left(\theta^{(2)}\right)^{\mathrm{t}} \nabla \mathbf{F}\left(\theta^{(2)}\right)\right]^{-1} \nabla \mathbf{F}\left(\theta^{(2)}\right)^{\mathrm{t}}\left[\eta-\mathbf{F}\left(\theta^{(2)}\right)\right]
$$

Cette opération est répétée jusqu'à ce que l'erreur quadratique (18) ou $\theta$ n'évoluent plus en valeur relative par rapport à une tolérance donnée. Cette tolérance est fixée par l'utilisateur de l'algorithme comme un critère d'arrêt.

L'algorithme de Gauss-Newton est très rapide, certainement le plus rapide des algorithmes itératifs pour la résolution des systèmes non-linéaires. Cependant, il nécessite le calcul du gradient des fonctions non-linéaires à chaque itération. Dans notre cas, le modèle (13) ne permet pas d'obtenir un gradient sous forme analytique simple. Nous sommes donc obligés de calculer la dérivée de chaque composante $F_{\imath}(x)$ de $\mathbf{F}(\theta)$ de façon approchée

$$
\frac{\partial F_{2}}{\partial x_{\jmath}}=\frac{F_{2}\left(x_{\jmath}+\delta x_{j}\right)-F_{2}\left(x_{\jmath}\right)}{\delta x_{\jmath}}
$$

où $x_{j}$ est l'une des variables du vecteur $\theta$ et $\delta x_{j}$ une petite variation en $x$. Cette approche risque d'engendrer des valeurs bruitées du gradient, ce qui est dû à la précision numérique limitée des calculs. La convergence devient alors incertaine.

3.3.2. Algorithme itératif proposé. - Au paragraphe 3.2., nous avons discuté de l'estimation du vecteur inconnu $\theta$ basée sur le modèle (14). Cette méthode repose sur une approximation linéaire de la relation inverse $\theta=\Phi\left(R_{\mathrm{n}}, X_{\mathrm{n}}\right)$. Supposons maintenant que l'on dispose déjà d'un modèle du type (14) et que l'on veuille l'affiner pour avoir une meilleure estimation de $\theta$. Pour ce faire, on peut reporter l'estimation courante $\left(\theta^{(2)}\right)$ dans le modèle (13) et créer ensuite une base de valeurs d'apprentissage pour procéder à la calibration du modèle (14). Ainsi, on obtient un nouveau modèle du type (14) et on peut faire un nouveau test de l'estimation. Cette procédure peut être réitérée jusqu'à ce que l'estimation de $\theta$ ne varie plus, ou que les valeurs de $R_{\mathrm{n}}$ et $X_{\mathrm{n}}$ prédites par le modèle (13) ne diffèrent pratiquement plus des valeurs mesurées. On obtient alors la meilleure estimation de $\theta$, ainsi que les meilleurs coefficients $c_{2 j}$ de la matrice $\mathbf{C}(15)$. Dans le cas où l'on doit contrôler plusieurs pièces dont les propriétés ne varient que faiblement autour des valeurs qui viennent d'être estimées, le nouveau modèle (14) pourrait être utilisé directement, sans recalibration, car l'approximation linéaire est suffisamment précise dans ce domaine de travail.

L'avantage principal de cette méthode réside dans la génération des valeurs de calibration du modèle (14) (voir paragraphe 3.2). En effet, lors de l'étape de calibration, on peut prendre des vecteurs $\theta=\left[\begin{array}{lll}d & \sigma & \mu_{r}\end{array}\right]^{t}$ avec une dynamique importante sur chaque composante $d, \sigma$ et $\mu_{\mathrm{r}}$. On linéarise ainsi grossièrement le modèle (13) par le modèle linéaire (14) dans un large domaine autour du point de fonctionnement correspondant. Ceci a deux intérêts : i) éviter de bâtir l'équation (13) sur le gradient local dès le départ car finalement celui-ci risque de n'avoir qu'un rapport lointain avec l'allure globale de (13), ii) dans le cas où le calcul numérique du gradient (22) n'est pas assez précis, l'approximation linéaire de l'équation (13), qui repose sur la méthode des moindres carrés est plus fiable. Au fur et à mesure que l'estimation s'améliore (l'erreur quadratique entre les valeurs prédites et mesurées de $R_{\mathrm{n}}$ et $X_{\mathrm{n}}$ devient plus petite), nous diminuons l'écart entre les valeurs de calibration de $d$, et $\sigma$ et $\mu_{\mathrm{r}}$. Quand cet écart est assez faible et devient comparable au pas de calcul du gradient dans l'équation (22), le modèle (14) redevient le développement de Taylor de l'équation (13) autour du point de fonctionnement courant. Nous retrouvons la méthode de Gauss-Newton.

En pratique, pour chaque paramètre $d, \sigma$ et $\mu_{\mathrm{r}}$, nous prenons trois valeurs de calibration qui sont $p_{1}=p_{0}(1-\lambda), p_{2}=p_{0}, p_{3}=p_{0}(1+\lambda)$, avec $0<\lambda<1$ et où $p$ indique n'importe lequel des paramètres. $\lambda$ possède une valeur relativement grande au départ et diminue avec l'erreur 
quadratique $\varepsilon$. Si l'on utilise une valeur différente de $\lambda$ pour chaque paramètre, on a un vecteur $\Lambda=\left[\begin{array}{lll}\lambda_{\mu_{\mathrm{r}}} & \lambda_{\sigma} & \lambda_{\mathrm{d}}\end{array}\right]^{\mathrm{t}}$.

3.3.3. Application aux cas réels. - Nous avons utilisé notre capteur pour déterminer les paramètres $\sigma, \mu_{\mathrm{r}}$ et $d$ de certaines cibles métalliques. Deux cas typiques sont discutés ici : cible en laiton (amagnétique, valeur nominale de $\sigma=14 \mathrm{MS} / \mathrm{m}$ ) et cible en acier XC38 (magnétique, valeur nominale de $\sigma=1 \mathrm{MS} / \mathrm{m}$, valeur nominale de $\mu_{\mathrm{r}}$ inconnue). La distance $d$ peut varier de $0,1 \mathrm{~mm}$ à $1,5 \mathrm{~mm}$.

3.3.3.1. - Initialisation de l'algorithme. - Les algorithmes itératifs sont sensibles à l'initialisation. Si un algorithme est initialisé trop loin de la solution, il convergera lentement, voire divergera. Nous avons testé l'algorithme avec plusieurs points d'initialisation. Il s'avère que notre algorithme converge encore bien pour des valeurs initiales très différentes des valeurs finales.

3.3.3.2. - Choix des fréquences de mesures. - Le choix des fréquences du courant d'excitation du capteur a été abordé au paragraphe 3.1. Pour le laiton, l'excitation mono-fréquence à 100 $\mathrm{kHz}$ suffit car $\mu_{\mathrm{r}}$ est égale à 1 , seules $\sigma$ et $d$ sont inconnues. Pour l'acier XC38, une deuxième fréquence d'excitation à $100 \mathrm{kHz}$ est nécessaire. Cependant, nous avons rajouté une autre fréquence d'excitation à $48 \mathrm{kHz}$ pour augmenter la complémentarité des informations. Nous avons vérifié la sensibilité du capteur par rapport à $\mu_{\mathrm{r}}, \sigma$, et $d$ aux fréquences choisies par simulation à l'aide du modèle (13).

3.3.3.3. - Choix du domaine d'approximation. - Le modèle (13) est utilisé pour construire une base de valeurs pour la calibration du modèle linéaire (14). Le choix du vecteur $\Lambda=$ $\left[\begin{array}{lll}\lambda_{\mu_{\mathrm{r}}} & \lambda_{\sigma} & \lambda_{d}\end{array}\right]^{\mathrm{t}}$ est très important pour cet algorithme. Il est pour l'instant heuristique mais dépend sûrement de la non-linéarité du modèle par rapport à chaque paramètre. En général, les composantes de $\Lambda$ sont grandes au départ (on considère en première approximation que le modèle (13) est linéaire sur un grand domaine). Elles seront diminuées ensuite si l'algorithme converge.

3.3.3.4. - Contraintes sur la solution. - Il arrive qu'au cours d'une itération, le modèle linéaire local donne une estimation absurde des paramètres recherchés, ce qui augmente l'erreur quadratique au lieu de la diminuer. Il existe deux remèdes à ce problème :

1) Borner le domaine de variation des paramètres : à chaque fois que les paramètres estimés dépassent ces bornes, les valeurs aux bornes sont utilisées pour ré-initialiser l'algorithme.

2) Introduire un frein : si la nouvelle estimation $\theta^{(\imath+1)}$ est mauvaise, on peut diminuer le pas de recherche en prenant une estimation modifiée

$$
\theta^{(\imath+1)^{\prime}}=\theta^{(\imath)}+\delta\left(\theta^{(\imath+1)}-\theta^{(\imath)}\right)
$$

avec $0<\delta \leq 1$. Si $\delta=1$, l'effet du frein disparaît.

3.3.3.5. - Simplification de l'algorithme. - Dans le cas de la détermination de 3 paramètres, il faut calculer 27 valeurs de $Z_{\mathbf{n}}$ à chaque fréquence d'excitation en étape de calibration du modèle (14) (chacun des paramètres $\mu_{\mathrm{r}}, \sigma$ et $d$ possède 3 valeurs, le nombre de combinaisons possibles de ces valeurs est $3^{3}$, soit 27 ). Si seules 2 fréquences d'excitation sont utilisées, il faut évaluer $Z_{\mathrm{n}} 54$ fois. Or, l'évaluation de $Z_{\mathrm{n}}$ est très coûteuse en temps de calcul. 


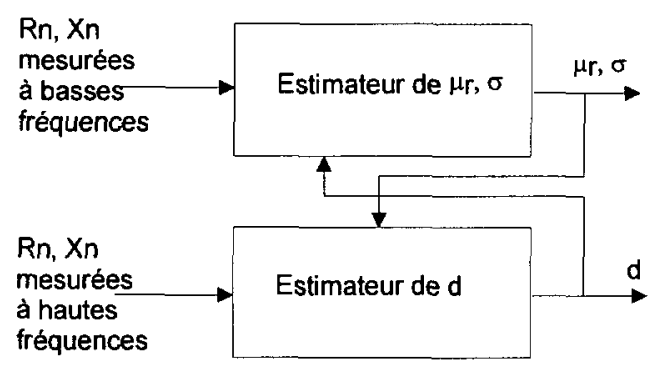

Fig. 8. - Schéma de l'algorithme itératif simplifié.

[Scheme of simplified iterative method.]

Pour diminuer le nombre d'évaluations du modèle, nous choisissons d'estimer $\mu_{\mathrm{r}}$ et $\sigma$ à un point de mesure à basse fréquence et seulement la distance $d$ à un point à haute fréquence. En effet, à haute fréquence, le capteur est surtout sensible à $d$ et peu sensible à $\mu_{\mathrm{r}}$ et $\sigma$ (Fig. 6c). Nous avons donc deux estimateurs linéaires dont l'un concerne $\left(\mu_{\mathrm{r}}, \sigma\right)$, et l'autre $d$. Dans le cas de l'utilisation de 2 fréquences d'excitation, le nombre d'évaluations du modèle est passé ainsi de 54 à 12 (9 pour la basse fréquence, 3 pour la haute fréquence). Pour l'estimateur "haute fréquence", bien qu'il faille calculer seulement $d$, nous rajoutons toutefois les variations en $\mu_{\mathrm{r}}$ et $\sigma$ dans le vecteur de calibration. Ainsi, l'estimateur pourra reconnaître les variations de $\mu_{\mathrm{r}}$ et $\sigma$, les "rejeter" et retenir seulement les variations de $d$.

Comme l'indique la figure 8, l'algorithme est initialisé avec une combinaison de valeurs de $d$, $\mu_{\mathrm{r}}$ et $\sigma$. L'estimateur "basse fréquence" donne des valeurs approchées de $\mu_{\mathrm{r}}$ et $\sigma$. Ensuite, en se servant de ces valeurs approchées et de la valeur initiale de $d$ pour déterminer un nouveau point de fonctionnement, l'estimateur "haute fréquence" calcule la nouvelle valeur de $d$. Ainsi se termine la première itération. L'itération suivante se déroule avec les valeurs approchées de $d, \mu_{\mathrm{r}}$ et $\sigma$ qui viennent d'être obtenues. L'erreur quadratique totale à chaque itération est la somme des erreurs quadratiques "partielles" commises pour chaque fréquence. Elle nous indique la convergence de l'algorithme alors que les erreurs "partielles" permettent de déterminer le vecteur $\Lambda$ adapté à chaque itération.

Dans le cas où $\mu_{\mathrm{r}}$ et $\sigma$ ne varient que faiblement, l'estimateur "basse fréquence" peut rester linéaire et peut être gardé constant durant l'inversion. Ce cas qui correspondrait, par exemple, à un contrôle de surface métallique permet d'économiser largement le temps de calcul.

3.3.3.6. - Précision de l'inversion. - Le problème inverse peut donner des solutions qui ne sont pas uniques. A cela, plusieurs raisons possibles :

1) Le modèle peut avoir une même valeur de sortie pour plusieurs combinaisons de paramètres d'entrée.

2) A cause des mesures bruitées, les estimations peuvent être instables.

3) L'algorithme est mauvais et ne converge pas vers la bonne solution.

La figure 9 illustre ces cas. On peut remarquer qu'il existe une ambiguité sur $R_{\mathrm{n}}$ par rapport à $\sigma$, mais non sur $X_{\mathrm{n}}$. Grâce à la combinaison de ces deux informations, nous avons pu éviter cette ambiguïté. Sur la figure $9 \mathrm{~b}$, on peut remarquer que quand $\frac{\partial X_{\mathrm{n}}}{\partial \sigma}$ est faible (capteur peu sensible à $\sigma$ ), une petite incertitude sur la mesure de $X_{\mathrm{n}}$ provoque une grande incertitude 

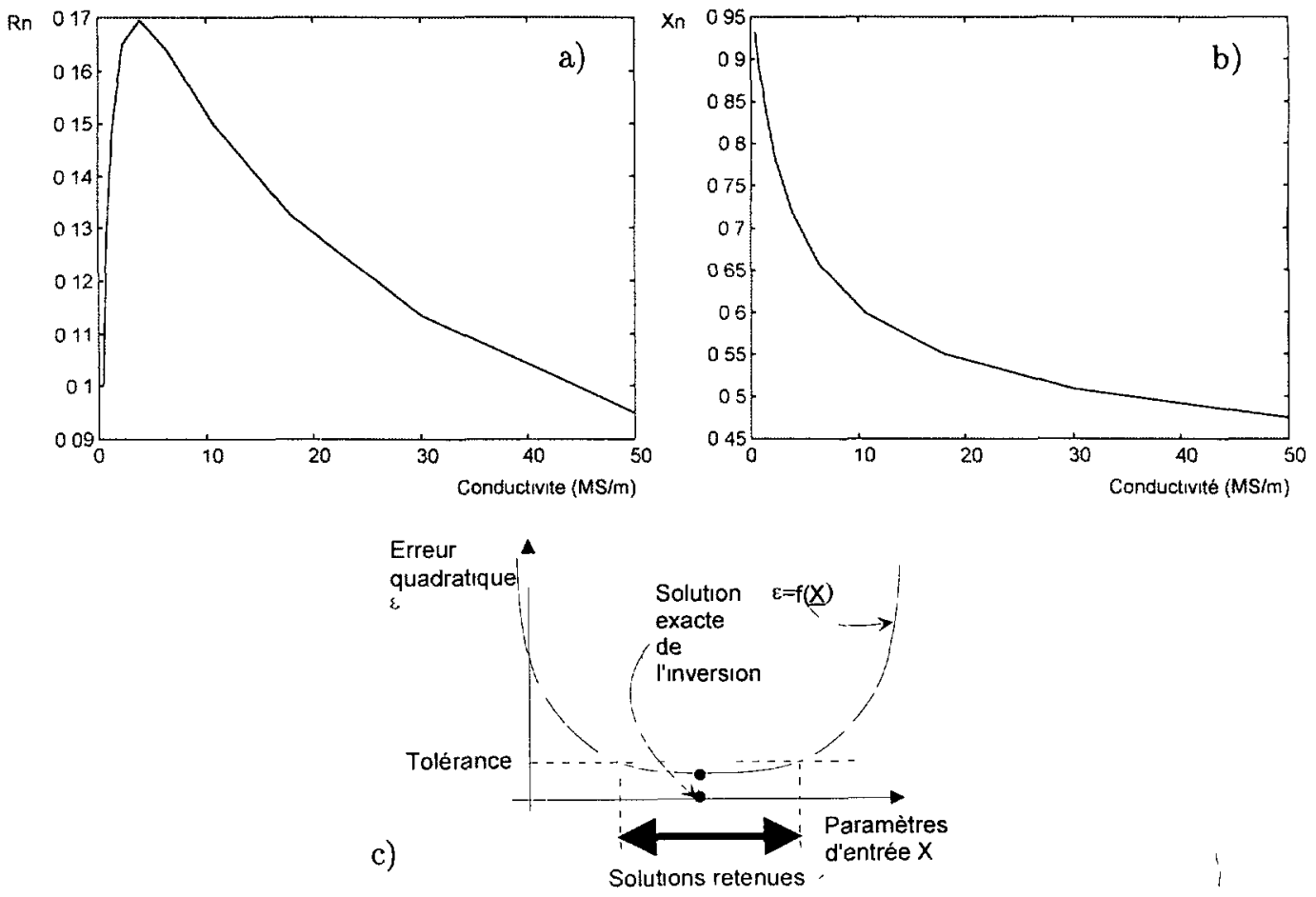

Fig. 9. - Cas d'inversion susceptibles de conduire à des résultats erronés : a) $R_{\mathbf{n}}$ présente une ambiguilté par rapport à $\sigma, \mathrm{b}$ ) Pour de grandes valeurs de $\sigma, \frac{\partial X_{\mathrm{n}}}{\partial \sigma}$, est très faible, c) Quand la tolérance sur le critère de convergence n'est pas assez faible, plusieurs solutions peuvent être retenues.

[Inversion cases which may lead to false solutions: a) $R_{\mathrm{n}}$ presents an ambiguity on $\sigma . \mathrm{b}$ ) $\frac{\partial X_{\mathrm{n}}}{\partial \sigma}$ is very weak for great values of $\sigma . \mathrm{c}$ ) When the tolerance on convergence criteria is not sufficiently small, many solutions may be retained.]

sur l'estimation de $\sigma$. Ce problème doit être résolu soit par le choix d'un autre capteur plus sensible, soit par le choix d'une autre fréquence du courant d'excitation, pour laquelle $\frac{\partial X_{n}}{\partial \sigma}$ est plus grande. Finalement, quand l'algorithme ne converge pas vers la bonne solution, ce phénomène est visible parce que les mesures et les prédictions du modèle correspondant aux paramètres estimés ne sont pas proches. L'utilisateur peut donc en prendre conscience et ajuster l'algorithme pour obtenir une convergence correcte.

Par ailleurs, il ne faut pas négliger l'influence des erreurs dues à la modélisation. Ces erreurs sont des origines différentes :

1) A basses fréquences, les mesures ne sont pas très précises car le phénomène d'induction est faible.

2) A hautes fréquences, l'influence des capacités parasites inter-spires n'est pas négligeable.

3) Les hypothèses faites en étape de modélisation ne sont pas totalement satisfaites (géométrie du bobinage imparfaite, pertes dans le circuit magnétique, etc.). 


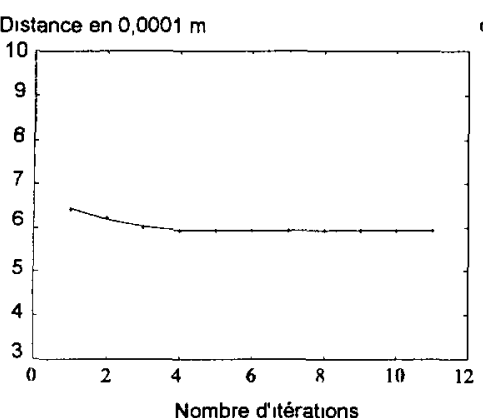

a)

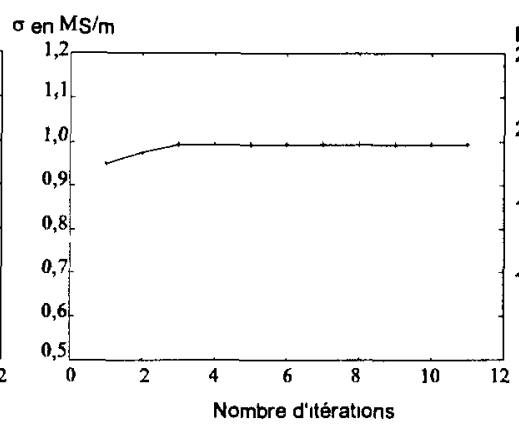

b)

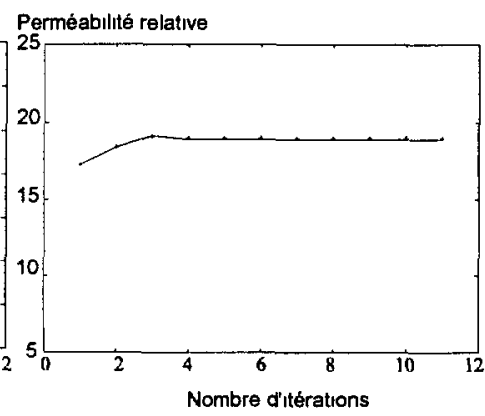

c)

Fig. 10. - Mesure sur l'acier XC38 avec excitation à trois fréquences (1 kHz, $48 \mathrm{kHz}, 100 \mathrm{kHz})$ par la méthode proposée (non simplifiée). a) Convergence de l'estimation de la distance capteur-cible (valeur exacte : $6 \times 10^{-4} \mathrm{~m}$ ). b) Convergence de l'estimation de la conductivité (valeur nominale : $1 \mathrm{MS} / \mathrm{m}$ ). c) Convergence de l'estimation de la perméabilité relative.

[Measuring XC38 steel using three excitation frequencies $(1 \mathrm{kHz}, 48 \mathrm{kHz}, 100 \mathrm{kHz})$ by proposed method (not simplified). a) Convergence of the estimation of lift-off (controlled value: $6 \times 10^{-4} \mathrm{~m}$ ). b) Convergence of the estimation of conductivity (nominal value: $1 \mathrm{MS} / \mathrm{m}$ ). c) Convergence of the estimation of permeability.]

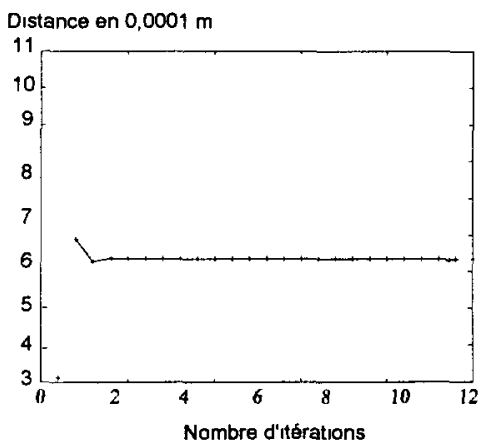

a)

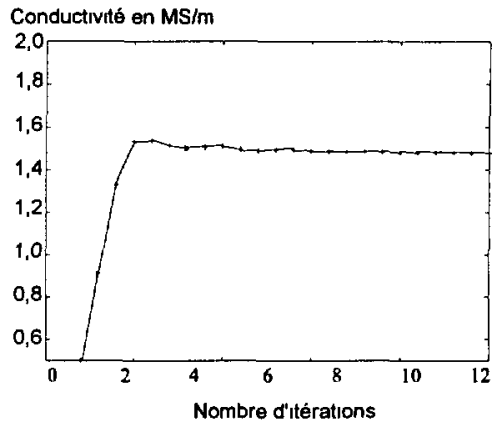

b)

Fig. 11. - Mesure sur le laiton avec excitation à une fréquence $(100 \mathrm{kHz})$. a) Convergence de l'estimation de la distance capteur-cible (valeur exacte : $6 \times 10^{-4} \mathrm{~m}$ ). b) Convergence de l'estimation de la conductivité (valeur nominale: $14 \mathrm{MS} / \mathrm{m}$ ).

[Measuring a copper alloy using single-frequency excitation $(100 \mathrm{kHz})$. a) Convergence of the estimation of lift-off (controlled value: $6 \times 10^{-4} \mathrm{~m}$ ). b) Convergence of the estimation of conductivity (nominal value: $14 \mathrm{MS} / \mathrm{m})$.

Pour remédier à ce problème, il faut éviter de faire des mesures à très hautes fréquences et donner une faible pondération aux mesures à basses fréquences. Par ailleurs, on peut essayer d'estimer l'erreur du $3^{\text {me }}$ type au préalable et de la corriger pendant l'inversion.

Nous présentons sur les figures 10 et 11 quelques exemples de la détermination des propriétés des métaux amagnétiques et magnétiques par cette méthode. 


\section{Conclusion}

La structure de CCF que nous venons de présenter possède certains avantages par rapport aux structures existantes, ne serait-ce que la possibilité d'adapter aisément sa géométrie à celle du problème traité, ce qui n'est pas tout à fait évident avec les structures de circuits magnétiques généralement utilisés, sauf usinages coûteux et délicats. De plus, la possibilité d'associer à cette structure un modèle analytique suffisamment précis permet à l'utilisateur une exploitation de données basée sur l'inversion d'un modèle sans avoir recours à une calibration expérimentale du CCF, qu'il serait d'ailleurs quasiment impossible à réaliser faute d'une connaissance préalable précise des conductivités et surtout des perméabilités des échantillons utilisés.

Nous avons proposé un algorithme dérivé de celui de Gauss-Newton, qui pourrait être très adapté à une exploitation industrielle "temps réel". Le choix de la fréquence d'excitation, question toujours d'actualité, a été discuté. Il a été démontré que l'on peut identifier les trois paramètres principaux d'une cible métallique $\left(\mu_{\mathrm{r}}, \sigma, d\right)$ en utilisant une excitation à deux fréquences au minimum. L'expérience peut être appliquée directement aux contrôles de haute précision de l'épaisseur d'un revêtement non-conducteur déposé sur une surface métallique de propriétés quelconques.

\section{Annexe}

ModÈle du CAPTEUR. - Quand le diamètre du bloc de ferrite au-dessus de la bobine est assez large par rapport au diamètre du bobinage, on peut l'assimiler à un plan infini et négliger les effets de bords. On trouve alors l'expression de l'impédance du capteur suivant la méthode exposée dans le paragraphe 2.2 :

$$
\begin{gathered}
Z=\frac{V}{i_{\text {exc }}}=\frac{j \omega \mu_{0} \mathrm{~N}^{2} \pi}{\left(r_{2}-r_{1}\right)^{2} l^{2}} \\
\int_{0}^{\infty} \frac{1}{\alpha^{5}}\left[\int_{\alpha r_{1}}^{\alpha r_{2}}\left(\alpha r_{0}\right) J_{1}\left(\alpha r_{0}\right) \mathrm{d}\left(\alpha r_{0}\right)\right]^{2}\left\{2 l+\frac{1}{\alpha}\left[\frac{\left(\mathrm{e}^{\alpha(l+d)}-\mathrm{e}^{\alpha d}\right) C_{1}+\left(\mathrm{e}^{-\alpha(l+d)}-\mathrm{e}^{-\alpha d}\right) C_{2}}{C_{3}}\right]\right\} \mathrm{d} \alpha
\end{gathered}
$$
avec

$\mathrm{N}:$ nombre de spires du capteur

$d:$ distance entre l'extrémité inférieure de la bobine et la cible

$\omega=2 \pi f, \mu_{0}=4 \pi \times 10^{7}$

$c:$ distance entre le plan de ferrite et la cible

$r_{1}$. rayon interne du bobinage,

$\alpha$ : variable de séparation ([10])

$r_{2}$ : rayon externe du bobinage,

$J_{1}(x)$ : fonction de Bessel de la première espèce d'ordre un.

$$
\begin{gathered}
C_{1}=\left(\alpha-\beta_{1}\right)\left(\alpha-\beta_{2}\right) \mathrm{e}^{-2 \alpha c-\alpha d}-\left(\alpha+\beta_{1}\right)\left(\alpha+\beta_{2}\right) \mathrm{e}^{-\alpha(l+d)} \\
+\left(\alpha-\beta_{1}\right)\left(\alpha+\beta_{2}\right) \mathrm{e}^{-2 \alpha c+\alpha(l+d)}-\left(\alpha-\beta_{1}\right)\left(\alpha+\beta_{2}\right) \mathrm{e}^{-2 \alpha c+\alpha d} \\
C_{2}=\left(\alpha-\beta_{1}\right)\left(\alpha-\beta_{2}\right) \mathrm{e}^{-\alpha d}-\left(\alpha+\beta_{1}\right)\left(\alpha+\beta_{2}\right) \mathrm{e}^{\alpha d}+\left(\alpha-\beta_{1}\right)\left(\alpha-\beta_{2}\right) \mathrm{e}^{(-2 \alpha c+\alpha(b+d)} \\
-\left(\alpha-\beta_{1}\right)\left(\alpha-\beta_{2}\right) \mathrm{e}^{-\alpha(l+d)} \\
C_{3}=\left(\alpha+\beta_{1}\right)\left(\alpha+\beta_{2}\right)-\left(\alpha-\beta_{1}\right)\left(\alpha-\beta_{2}\right) \mathrm{e}^{-2 \alpha c}
\end{gathered}
$$

où $\beta_{\imath}=\frac{\mu_{0}}{\mu_{2}} \sqrt{\alpha+j \omega \mu_{2} \sigma_{2}}, \mu_{\imath}$ et $\sigma_{\imath}$ sont la perméabilité (absolue) et la conductivité du milieu examiné, $i=1 . .4$ ( $i=1$ : ferrite, $i=2,3:$ air, $i=4:$ cible). 
En général, la perméabilité des matériaux varie en fonction du champ magnétique externe. Dans notre cas, puisque le champ magnétique créé par le capteur est très faible et alternatif de surcroît, la perméabilité dont nous discutons ici correspond au début de la courbe de magnétisation et est considérée comme constante (régime petit signaux).

\section{Bibliographie}

[1] Aknin P., Algorithme itératifs pour l'inversion d'un modèle non linéaire de multicapteur à courants de Foucault, Thèse de Doctorat (Université Paris Sud, Centre d'Orsay, 1990).

[2] Antoniadis A., Berruyer J. et Carmona R., Régression non linéaire et applications, Econ. (1993).

[3] Beissner R.E., Theory of Eddy-Current Characterization of Magnetic Conductors, in Proc. 3rd Int. Symposium., Non destructive Characterization of Materials Saarbrück, October 1988, P. Höller et al. Eds (Springer-Verlag, 1990) pp. 541-548.

[4] Bowler J.R., Sabbagh L.D. et Sabbagh H.A., A Theoretical and Computational Model of EddyCurrent Probes Incorporating Volume Integral and Conjugate Gradient Methods, IEEE Trans. Magn. 25 (1989).

[5] Cheng C., Dodd C.V. et Deeds W.E., General Analysis of Propbe Coils Near Stratified Conductors, Int. J. Non destr. Test. 3 (1971) 109-130.

[6] Deeds W.E. et Dodd C.V., Determination of Multiple Properties with Multiple Eddy Current Measurements, Int. Adv. Nondestr. Test. 9 W.J. McGonnagle, Gordon and Breach Ed. (New York, 1981) pp. 317-333.

[7] Dodd C.V., Deeds W.E. et Luquire, Integral Solutions to Some Eddy-Current Problems, Inter. J. Nondestr. Test. 1 (1969/1970) 29-90.

[8] Dufour I., Contribution à l'évolution des procédés de contrôle non destructif par capteurs inductifs : application au cas des matériaux composites carbones, Thèse de Doctorat (Université Paris Sud, Centre d'Orsay, 1993).

[9] Jacob D., Principe de la mesure simultanée de distance et d'épaisseur de dépôts métalliques par capteurs à courants de Foucault : conception et réalisation d'un dispositif, Thèse de Doctorat (Université Paris Sud, Centre d'Orsay, 1988).

[10] Panofsky W.K.H et Phillips M., Classical Electricity and Magnetism (Addison-Wesley, 1972).

[11] Placko D., Dispositif d'analyse de profil utilisant des capteurs à courants de Foucault, Thèse du 3è cycle (Université Paris Sud, Centre d'Orsay, 1984).

[12] Placko D., Contribution à la conception de capteurs inductifs pour la robotique industrielle, Diplôme d'habilitation à diriger des recherches en sciences (Université Paris Sud, Centre d'Orsay, 1990).

[13] Placko D., Aknin P. et Clergeot, Algorithme itératif séquentiel pour l'inversion d'un opérateur. Application à la restauration des images fournies par un multicapteur à courants de Foucault, Traitement du signal, 7 (1990) pp. 499-513.

[14] Press W.H., Flannery B.P., Teukolsky S. A. et Vettrling W. T., Numerical Recipes: The Art of ScientificComputing (Cambridge University Press, 1989).

[15] Walter E. et Pronzato L., Identification des Modèles Paramétriques à partir de Données Expérimentales, Laboratoire des Signaux et Systèmes, CNRS-Ecole Supérieure d'Electricité, Plateau de Moulon, 91192 Gif-surYvette Cedex, France (Masson Ed., 1994).

[16] Weaver J.T., The General Theory of Electromagnetic Induction in a Conducting Half-Space, Geophys. J. R. astron. Soc. 22 (1970) 83-70. 